\title{
Atypical Presentation of Lichen Planopilaris: Presentation of Two Cases and Review
}

\author{
Nwanneka Okwundu ${ }^{1 *}$, Felicia Ekpo ${ }^{2}$, Jessica Ghaferri ${ }^{2}$, David Fivenson ${ }^{2}$ \\ ${ }^{1}$ University of Utah Department of Dermatology, Salt-lake City, Utah, USA \\ ${ }^{2}$ St Joseph Mercy Hospital Dermatology Residency Program, Ann Arbor, Michigan, USA
}

Received: February 07, 2020; Accepted: February 23, 2020; Published: March 16, 2020

*Corresponding author: Nwanneka Okwundu,University of Utah Department of Dermatology, Salt-lake City, Utah, USA.

E-mail: pishgouym951@mums.ac.ir

\begin{abstract}
Lichen Planopilaris (LPP) is an uncommon scalp disorder of unknown etiology and prevalence. It is thought to be an autoimmune process triggered by unknown genetic and/or environmental factors that attack hair follicles of the scalp. LPP has been reported to mimic or present in association with various autoimmune diseases and immunomodulatory therapies. We present two atypical case of LPP in Caucasian patients; the first is a patient with generalized pruritus, skin eruptions, and scalp hair loss. Biopsy of the lesions revealed exocytosis of atypical lymphocytes at the Dermo-Epidermal Junction (DEJ) and formation of small Pautrier's microabscesses in the interfollicular epidermis as well as a robust lymphocytic inflammatory infiltrate with destruction of perifollicular appendages. This led us to the diagnosis of LPP-like Folliculotropic Mycosis Fungoides (FMF). The second case is a patient with a history of Systemic Lupus Erythematosus (SLE) who presented clinically with perifollicular erythema and alopecic patches, with loss of follicular ostia on the frontal and vertex scalp and evidence of follicular tufting. Histologically, she had decreased number of follicles with a peri-infundibular lymphocytic infiltrate and vacuolization at the Dermoepidermal Junction (DEJ) and there was also thickening of the basement membrane highlighted with a periodic acidic Schiff stain. This led to a diagnosis of SLE/LPP overlap.

Keywords: Lichen Planopilaris (LPP); Cicatricial; Alopecia; Frontal Fibrosing Alopecia (FFA), Folliculotropic Mycosis Fungoides (FMF), Lichen Planus (LP).
\end{abstract}

\section{Introduction}

Lichen Planopilaris (LPP) is a rare progressive form of scarring (cicatricial) alopecia [1]. It is also considered a lymphocytic cicatricial alopecia characterized by follicular hyperkeratosis, perifollicular erythema, and loss of follicular orifices[2-4].The annual incidence rate varied from $1.15 \%$ to $7.59 \%$ in a survey conducted by 4 medical centers. The age of onset for LPP has been found to be between 25 and 70 years and the most common reported symptoms of patients with LPP are; increase in shedding, pruritus, scale, and scalp tenderness [5].

The exact pathogenesis of LPP remains unclear; however, it is thought to be due to an autoimmune, cell-mediated cytotoxic immune reaction against follicular antigens $[3,6]$.Some consider LPP to be a follicular variant of lichen planus because its pathogenesis is similar to that of lichen planus. Since LPP is also thought to have an autoimmune pathogenesis, it may have a possible relationship to other autoimmune diseases [2,3].

In addition to having an autoimmune pathogenesis, LPP has also been found to occur in association with or mimic other autoimmune and non-autoimmune disorders. However, studies about its autoimmune and other co-morbid conditions are limited. It is more commonly associated with autoimmune disorders such as sjogren's syndrome and lupus erythematosus [7,8]. Nonautoimmune co-morbid conditions of LPPinclude hyperlipidemia, metabolic syndrome, hypothyroidism, anxiety, and depression [9-12]. In addition to its association to autoimmune and other comorbid condition, LPP interestingly continues to be discovered in atypical situations that have not been reported in the past. We present two atypical cases of LPP.

\section{Case1}

A 69-year-old female presented with a pruritic rash that started fourteen months prior to her initial dermatology visit. Her symptoms began with generalized itching, after which she noticed a skin eruption on her bilateral breasts and groin. This was unresponsive to triamcinolone cream, fexofenadine, and cetirizine. Chest x-ray and initial laboratory tests were unremarkable. Anti-nuclear antibody, vitamin D, bile acids, rheumatoid factor, C-reactive protein, insulin and reproductive hormones such as follicle stimulating hormone, luteinizing hormone, progesterone, and estrogen which were normal. Allergy testing was also negative for environmental or chemical allergens. As the pruritus worsened and spread to her scalp, she reported gradual scalp hair loss. This hair loss started seven months prior to presentation resulting in the patient having to wear a wig.

Physical exam revealed perifollicular erythema, follicular tufting, loss of follicular ostia, and alopecic patches with no evidence of hair regrowth on the vertex scalp. Hair pull test was negative. Dermatologic exam of the body revealed generalized atrophic poikilodermatous patches with a background of mottled erythema on the chest, upper and lower extremities, abdomen, and flanks (figure 1).

A skin biopsy was performed from the vertex scalp, 


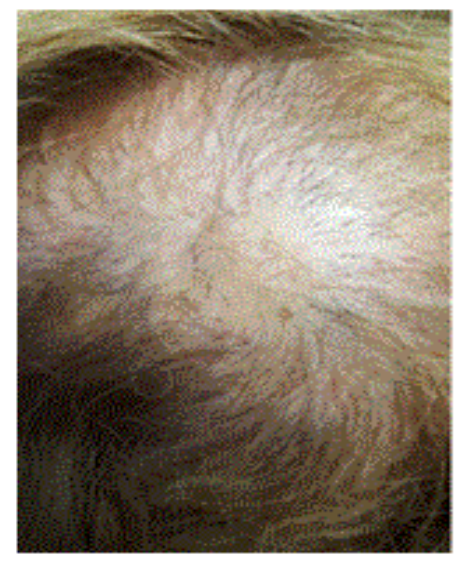

Figure 1: Perifollicular erythema and tufting with loss of follicular ostia on vertex scalp.

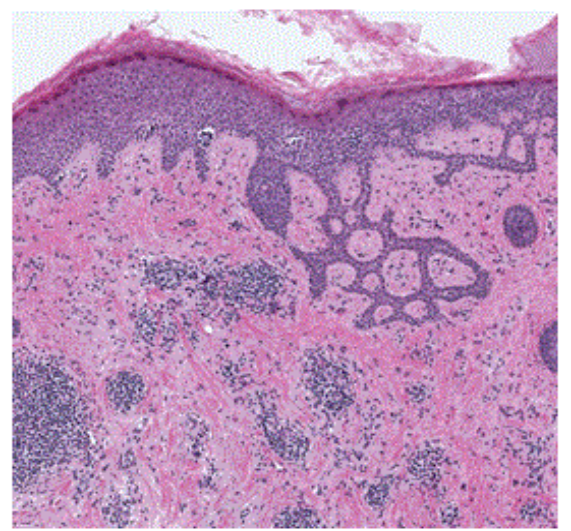

Figure 2: Exocytosis of atypical lymphocytes at the DEJ and formation Pautrier's microabscesses with a sparse lichenoid infiltrate at the Dermoepidermal Junction (DEJ).

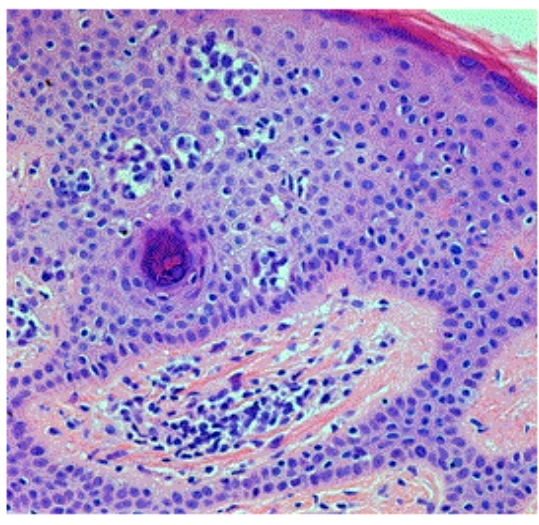

Figure 3: Robust lymphocytic inflammatory infiltrate with destruction of perifollicular appendages, perifollicular fibrosis and polytrichia showing evidence of scarring. which revealed exocytosis of atypical lymphocytes at the Dermoepidermal Junction (DEJ) and formation of small Pautrier's microabscesses in the interfollicular epidermis (figure 2). A robust lymphocytic inflammatory infiltrate with destruction of perifollicular appendages were also noted with remaining follicles showed polytrichia with perifollicular fibrosis and follicular plugging (figure 3). Immunohistochemical studies revealed a predominance of $\mathrm{CD} 3+$ and $\mathrm{CD} 4+\mathrm{T}$ cells and a 10:1 ratio of $\mathrm{CD} 4: \mathrm{CD} 8$ positivity. The histology was interpreted as Folliculotropic Mycosis Fungoides (FMF) presenting clinically as LPP. Biopsy from the skin eruption also showed Mycosis Fungoides (MF) with similar immunohistochemistry and a positive T-cell receptor gene rearrangement. Treatment with clobetasol foam, narrowband UVB, and acitretin resulted in partial improvement of our patient's skin eruption, pruritus, and alopecia.

\section{Case2}

A 63-year-old female presented with a four-year history of dull scalp pain and hair loss on her scalp and eyebrows diagnosed by the association of positive ANA, fatigue, and inflammatory arthritis. Past medical history was significant for SLE. The patient's lupus was controlled with mycophenolate mofetil $1000 \mathrm{mg}$ daily, topical steroids, COQ10 $250 \mathrm{mg}$ daily, and hydroxychloroquine $200 \mathrm{mg}$ twice a day, but hair loss had been progressive over the preceding six months.

Physical exam of the scalp revealed perifollicular erythema and alopecic patches with loss of follicular ostia on the frontal and vertex scalp and evidence of follicular tufting. She also had a prominent malar facial rash. Follicular hyperkeratosis was observed along the frontal hairline (figure 4). Examination of the eyebrows showed atrophy and thinning bilaterally.

Labs revealed a positive ANA of 1:1280 and elevated creatine phosphokinase (CPK) of 387 and 271 taken 3 months apart. Additional labs performed were normal, including urinalysis, anti-deoxyribonucleic acid (DNA), CBC, LFTs, erythrocyte sedimentation rate (ESR), CRP, thyroid stimulating hormones (TSH), vitamin $\mathrm{D}$, vitamin $\mathrm{B}_{12}$, folate, immunoglobulins, and complement levels.

Biopsy from the left temporal scalp revealed a decreased number of follicles with a peri-infundibular lymphocytic infiltrate and vacuolization at the DEJ (figure 5). There was thickening of the basement membrane highlighted with a periodic acidic schiff (PAS) stain (figure 6). The histology was suggestive of LPP with an overlap of SLE given the patient's clinical examination and history. The patient's hair loss improved with initiation of oral finasteride $5 \mathrm{mg}$ daily, oral methotrexate weekly, topical clobetasol, minoxidil foam, and discontinuation of mycophenolate mofetil. 


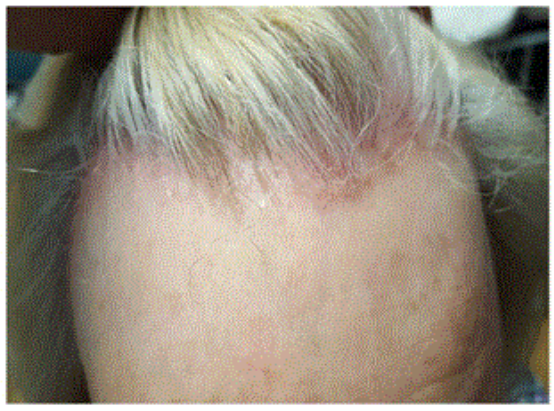

Figure 4: Perifollicular erythema with loss of follicular ostia along frontal hairline.

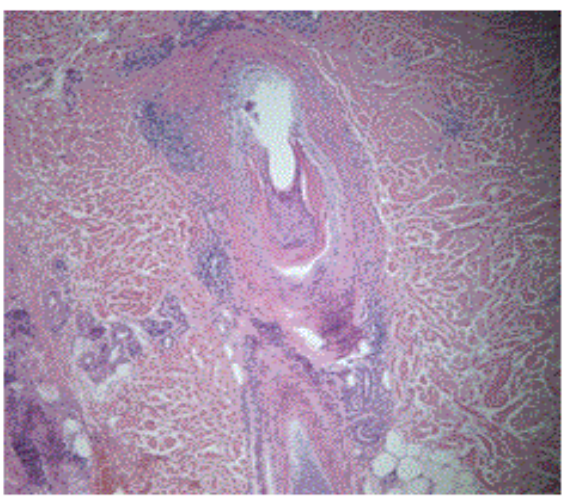

Figure 5: Peri-infundibular lymphocytic infiltrate and vacuolization

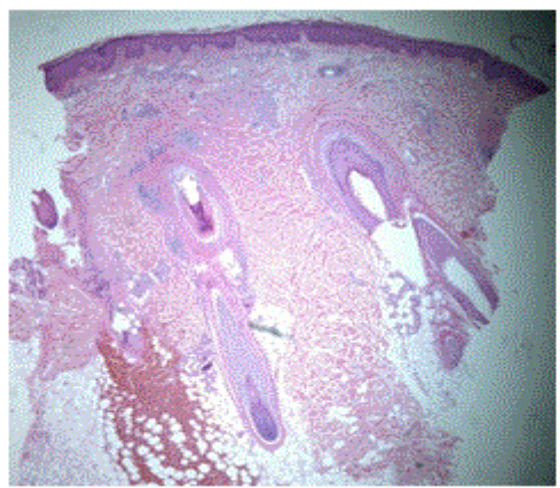

Figure 6: Peri-infundibular lymphocytic infiltrate and vacuolization with thickening of the basement membrane

\section{Discussion}

Lichen Planopilaris (LPP) is an inflammatory scalp disorder that is characterized by perifollicular erythema, follicular hyperkeratosis, and scarring alopecia [13]. The permanent loss of hair is postulated to emanate from the site in which inflammation occurs. Inflammatory infiltrate in LPP is mainly concentrated around the follicular infundibulum and isthmus, unlike non-scarring forms of hair loss, e.g. alopecia areata, in which inflammation is mostly concentrated around the follicular bulb. The follicular "bulge" which is a site of pluripotent stem cells, involved in the regeneration of the lower portion of the hair follicle during follicular cycling is within the immunologic target for LPP. Damage to the bulge region is postulated to account for the development of scarring alopecia [14]. Pseuodopelade of Brocq is used to describe the non-inflammatory end stage patchy cicatricial alopecia that may be seen in LPP [15].

There are three variants of LPP that can be distinguished based on their clinical patterns and areas of involvement. These variants include classic LPP, FFA, and Graham-Little-PiccardiLasseur Syndrome (GLPLS)[16,17]. Classic LPP usually involves the vertex and parietal areas of the scalp. The second variant of LPP, FFA, is characterized by fronto-temporal band-like loss of both terminal and vellus hairs. Small numbers of isolated hairs are often spared within the band of alopecia, resulting in a finding called the "lonely hair sign". Similar to classic LPP, erythematous and hyperkeratotic follicles are often found at the peripheral areas of alopecia. Hypopigmentation may be evident in the affected areas. Pruritus or trichodynia are occasionally reported. FFA may involve other scalp sites, such as the periauricular, parietal, or occipital areas [18].

Case 1 highlights LPP overlapping with FMF. FMF is a rare, but distinct variant of Cutaneous T-cell Lymphoma (CTCL). FMF usually involves the head and neck in comparison to classic MF, which presents in the typical bathing suit distribution. The morphologic features seen in FMF include erythematous papules and plaques with follicular prominence, with or without alopecia. It also includes comedonal acneiform (unlike some of the features on presentation of our first case), nodular, prurigo-like and cystic lesions, as well as alopecic patches with or without scarring. The differential diagnosis of LPP-like FMF include long-standing alopecia areata, seborrheic dermatitis, chronic telogen effluvium, and idiopathic follicular mucinosis.

Secondary alopecia mucinosa seen in older individuals which many now consider to be mycosis fungoides has a lot of similar features that can be seen in LPP overlapping with FMF [19]. It usually presents with several erythematous papules and plaques mainly over the head and face regions, as can be seen in LPP. However, we ruled out follicular mucinosis because it usually presents histologically with accumulation of mucin within the follicular infundibula unlike LPP or FMF $[20,21]$.

FMF could simulate a variety of inflammatory conditions that affect the follicular unit, including scarring alopecia. FMF may mimic the clinical presentation of Lichen Planopilaris (LPP) or keratosis pilaris. Histologic differential diagnoses include nodulocystic acne, eosinophilic or suppurative folliculitis, granulomatous dermatitis, or discoid lupus [22]. Our patient had clinical features of LPP but histologic findings of FMF.

Case two illustrates an overlap between LPP and SLE, a rare presentation of the Lichen Planus/ Lupus Erythematosus(LE/LP) overlap syndrome. Our patient had a history of SLE with positive ANA, and other systemic symptoms like fatigue and inflammatory arthritis, prior to the manifestation of alopecia and other LPP symptoms. Lichen planopilaris is thought to be a follicular 
variant of Lichen planus due to their similar pathophysiologic mechanism [2]. There are very few descriptions of LPP and Lupus Erythematosus (LE) coexisting together, with the discoid variant of LE being the most common association seen in the LE/LP overlap syndrome [8].

The other dilemma associated with the diagnosis of this overlap is that Discoid Lupus Erythematosus (DLE) may be indistinguishable clinically from LPP and biopsy is usually needed to prove the diagnosis. Histopathological changes of early DLE and LPP are different and characteristic. However, in the late stages, features are apparently difficult to differentiate. One helpful clinical distinguishing clue is that in contrast to DLE LPP presents with less dyspigmentation, a more intense pruritus, and perifollicular erythema as seen in our patient's clinical presentation [5,23]. Another helpful clinical clue is the presence of acuminate perifollicular keratotic plugs on the periphery of the alopecic patches as seen in our patient which is unlike the follicular plugging seen on the center of the alopecic patches in scalp DLE [24].

LPP and LE are said to have a common background and their co-existence may not be a coincidence [25]. The differential diagnosis for LPP with an SLE overlap includes cutaneous and discoid lupus erythematosus, seborrheic dermatitis, rosacea, lupus vulgaris, sarcoidosis, long-standing traction alopecia, androgenetic alopecia, keratosis follicularis spinulosa decalvans, pseudopelade of Brocq, alopecia mucinosa, familial high frontal hairline, and central cicatricial scarring alopecia [7].

\section{Conclusion}

We present two atypical cases of LPP with the first being an LPP-like presentation of FMF, and the second case is an SLE/LPP overlap form of the LE/LP syndrome. Lichen planopilaris remains an interesting disease mimicker and its etiology is multifactorial. LPP has also been associated with an increased risk of concomitant diseases such as hypothyroidism,hypertension, and metabolicdisorders. Associations continue to arise, such as in our patient with SLE and a mimicking situation as seen in our case of the LPP-like FMF. We suggest clinicians maintain a high index of suspicion for associated diseases in the presentation of a new LPP pattern. Early initiation of treatment is paramount to preserve the remaining unaffected hair follicles and may be done in tandem with underlying disease as we have demonstrated.

\section{References}

1. Alahmari L, Almesned R, Alhumidi A, Alkhalifah A. Lichen planopilaris with Koebner phenomenon. JAAD Case Reports. 2018;4(8):848-850. doi:10.1016/j.jdcr.2018.05.009

2. Bolduc C, Sperling LC, Shapiro J. Primary cicatricial alopecia: Lymphocytic primary cicatricial alopecias, including chronic cutaneous lupus erythematosus, lichen planopilaris, frontal fibrosing alopecia, and Graham-Little syndrome.J Am Acad Dermatol. 2016;75(6):10811099.doi: 10.1016/j.jaad.2014.09.058

3. Conic RR, Piliang M, Bergfeld W, Atanaskova Mesinkovska N. Association of Lichen Planopilaris with Dyslipidemia. JAMA
Dermatology. 2018;154(9):1088-1089.

doi:10.1001/ jamadermatol.2018.1749

4. Saceda D, Fernández $\mathrm{P}$, Fonda $\mathrm{P}$, Pindado $\mathrm{C}$, Moreno OM, VañóKarger. Clinical Description of Frontal Fibrosing Alopecia with Concomitant Lichen Planopilaris, 2018;4:105-107.

5. Tan E, Martinka M, Ball N, Shapiro J. Primary cicatricial alopecias: clinicopathology of 112 cases. Journal of the American Academy of Dermatology. 2004;50(1):25-32. doi: 10.1016/j.jaad.2003.04.001

6. Chieregato C, Zini A, Barba A, Magnanini M, Rosina P. Lichen planopilaris: report of 30 cases and review of the literature. Int J Dermatol. 2003;42(5):342- 345. doi:10.1046/j.1365-4362.2003.01695.x

7. Furlan KC, Kakizaki P, Chartuni JC, Valente NY. Frontal fibrosing alopecia in association with Sjögrens syndrome: More than a simple coincidence. Anais Brasileiros De Dermatologia. 2016;91(5 suppl 1):14-16. doi:10.1590/abd1806-4841.20164526

8. Contin LA, Elisa Marques, Noriega L. Frontal fibrosing alopecia coexisting with lupus erythematosus: Poor Response to Hydroxychloroquine. Skin Appendage Disorders. 2016;2(3-4):162166. doi:10.1159/000452925.

9. Garcia PMJ, LlorentePS, Seoane RJM, Berasaluce MJ, García JM. Thyroid disease and oral lichen planus as comorbidity: A prospective casecontrol study. Dermatology. 2016;232(2):214-219.

10. Hirota SK, Moreno RA, Dos Santos, Seo J, Migliari DA. Psychological profile (anxiety and depression) in patients with oral lichen planus: A controlled study. Minerva Stomatol. 2013;62(3):51-56.

11. LópezJP, Parra F, PonsFA. Association of autoimmune diseases with oral lichen planus: A cross-sectional, clinical study. J Eur Acad Dermatol Venereol. 2014;28(7):895-899.

12. Lai YC, Yew YW, Schwartz RA. Lichen planus and dyslipidemia: A systematic review and meta-analysis of observational studies. Int J Dermatol. 2016;55(5):295-304.

13. Mardones F, Shapiro J. Lichen planopilaris in a Latin American (Chilean) population: demographics, clinical profile and treatment experience. Clinical and Experimental Dermatology. 2017; 42(7):755759. doi: 10.1111/ced.13203.

14. Chang C, Sain D, Cho BK. Hydroxychloroquine and Lichen planopilaris Activity Index Scoring System. J Am Acd Dermatol. 2010;62(3):387392.

15. Ross EK, Tan E, Shapiro J. Update on primary cicatricial alopecias. Journal of the American Academy of Dermatology. 2005;53(1):1-37. doi: 10.1016/j.jaad.2004.06.015

16. Olsen EA, Bergfeld WF, Cotsarelis G. Summary of North American Hair Research Society (NAHRS)-sponsored Workshop on cicatricial alopecia, Duke University Medical Center, February 10 and 11, 2001. J Am Acad Dermatol. 2003;48(1):103-110.

17. Meinhard J, Stroux A, Lünnemann L. Lichen planopilaris: epidemiology and prevalence of subtypes-a retrospective analysis in 104 patients. J Dtsch Dermatol Ges. 2014;12(3):229-235. doi: 10.1111/ddg.12264.

18. Glick B, Singer T, Wiener A. Hydroxychloroquine and lichen planopilaris: Efficacy and introduction of Lichen planopilaris activity index scoring system. Yearbook of Dermatology and Dermatologic Surgery. 2011;191-192.

19. Joshi R, Gopalani V. Alopecia mucinosa responding to antileprosy treatment: Are we missing something? Indian Journal of Dermatology. 
2013;58(3):227. doi:10.4103/0019-5154.110834

20. Hempstead RW, Ackerman AB. Follicular mucinosis: A reaction pattern in follicular epithelium. Am J Dermatopathol. 1985;7(3):245-257.

21. Elder DE, Elenitsas R, Johnson BL Jr, Murphy GF, Xu X. Lever's Histopathology of the skin.10th ed. Philadelphia: Wolters Kluwer / Lippincott Williams \& Wilkins. Follicular Mucinosis and Alopecia Mucinosa. Inflammatory diseases of hair follicles sweat glands and cartilage. 2009;pp:467-468.

22. Gerami P, Rosen S, Boone S, Guitart J. Folliculotropic Mycosis Fungoides. An aggressive variant of cutaneous T cell Lymphoma. Arch Dermatology. 2008;144(6):738-746.
23. Moghadam S, Franks AG. Autoimmune disease and hair loss. Dermatol Clin. 2013;31(1):75-91. doi:10.1016/j. det.2012.08.008

24. Semble AL, McMichael AJ. Hair loss in patients with skin of color. Semin Cutan Med Surg. 2015;34(2):81-88. doi:10.12788/j.sder.2015.0145

25. Nascimento LL, Milvia E, Vasconcellos MR. Coexistence of chronic cutaneous lupus erythematosus and frontal fibrosing alopecia. Anais Brasileiros De Dermatologia. 2018;93(2):274-276. doi:10.1590/ abd1806-4841.20186992. 